Mini Review

\title{
Role of Colchicine in Management of COVID-19?
}

\author{
Kholoud Mohamed Elawady Elhefnawy Elshiwy ${ }^{1}$, Ghada Essam El-Din Amin ${ }^{1,2}$, Mohamed Nazmy ${ }^{3}$, Rasha Samir \\ 3, Mohamed Farouk Allam 1,4*0
}

How to cite this paper: Elshiwy, K. M. E. E., Amin, G. E. E., Farris, M. N., Samir, R., and Allam, M. F. (2021). Role of Colchicine in Management of COVID-19?. Global Journal of Epidemiology and Infectious Disease, 2(1), 1-3. Retrieved from https://www.scipublications.com/jou rnal/index.php/gjeid/article/view/17 7

Received: November 09, 2021 Accepted: December 15, 2021 Published: December 16, 2021

Copyright: (C) 2022 by the authors. Submitted for possible open access publication under the terms and conditions of the Creative Commons Attribution (CC BY) license (http://creativecommons.org/licenses /by/4.0/).

\author{
${ }^{1}$ Department of Family Medicine, Faculty of Medicine, Ain Shams University, Cairo, Egypt \\ 2 Department of Community, Environmental and Occupational Medicine, Faculty of Medicine, Ain Shams \\ University, Cairo, Egypt \\ ${ }^{3}$ Department of Internal Medicine, Faculty of Medicine, Ain Shams University, Cairo, Egypt \\ ${ }^{4}$ Department of Preventive Medicine and Public Health, School of Medicine, University of Cordoba, Cordoba \\ 14004, Spain \\ *Correspondence: farouk.allam@med.asu.edu.eg
}

\begin{abstract}
CoV-2 disease 2019 (COVID-19) pandemic has exerted a great burden on the health and economic systems worldwide. One of the most important factors that affect the severity and prognosis of COVID-19 is the occurrence of hyperstimulation of the immune system resulting in "cytokine storm". Similar to SARS-CoV, an intracellular complex called nod like receptor family pyrin domain containing 3 (NLRP3) inflammasome was found to be activated by SARS-CoV-2, then in turn stimulates several interleukins and initiates the inflammatory cascade. In addition, other inflammatory mediators such as interferons e.g., IFN- $\alpha$, and IFN- $\gamma$, interleukins e.g., IL-1 $\beta$, IL-12, IL-18, tumor necrosis factor- $\alpha$ (TNF- $\alpha$ ), and chemokines e.g., CCL2, CXCL10 have been reported in severely ill patients. These mediators represent the corner stone in developing cytokine storm that results in uncontrolled systemic inflammatory reaction with subsequent acute respiratory distress syndrome (ARDS), multiple organ failure and eventually death. Based on its anti-inflammatory effects, colchicine has also gained attention to be utilized in the management of COVID-19 patient. Colchicine exerts its anti-inflammatory effect through inhibition of formation of microtubules which is considered an essential step in several cellular processes such as division, signalling, and migration. Also, colchicine affects the cytokine cascade by inhibiting IL-1 $\beta$ leading to reduction in neutrophils recruitment, free radicles production and inflammasome stimulation. This raises the concerns about the effectiveness of colchicine in COVID-19 treatment and the possibility of providing an improvement of the clinical course of the disease.
\end{abstract}

Keywords: Coronavirus, COVID-19, Colchicine, Management, Review

\section{Mini Review}

CoV-2 disease 2019 (COVID-19) pandemic has exerted a great burden on the health and economic systems worldwide. COVID-19 is caused by SARS-CoV-2 which belongs to coronaviruses family that also includes SARS-CoV and MERS-CoV [1].

In China, SARS-CoV caused outbreaks of severe respiratory distress syndrome in 2002 and 2003 [2]. While in 2012, outbreaks of severe respiratory distress syndrome have been reported in the Middle East and was caused by MERS-CoV [3]. All coronaviruses including SARS-CoV-2 are characterized by the presence of S proteins in the viral envelope that surrounds the nucleocapsid formed of RNA and phosphorylated nucleocapsid protein [4]. S proteins are responsible for direct binding between the viral particles and host cell membrane through interaction with angiotensin converting enzyme 2 (ACE2) receptor, which is found in different tissues such as lung, kidney, heart, and intestine [5]. This can contribute to affection of COVID-19 to multiple body systems and clinical 
presentation ranges from mild symptoms to severe cases of pneumonia, acute organ failure such as kidney, and ischemic events [6]. One of the most important factors that affect the severity and prognosis of COVID-19 is the occurrence of hyperstimulation of the immune system resulting in "cytokine storm" [7]. Similar to SARS-CoV, an intracellular complex called nod like receptor family pyrin domain containing 3 (NLRP3) inflammasome was found to be activated by SARS-CoV-2, then in turn stimulates several interleukins and initiates the inflammatory cascade [8]. Also, interleukin-6 (IL-6) has a role in inducing cytokine storm. IL- 6 is produced by different immune cells under stimulation by tumor necrosis factor- $\alpha$ (TNF- $\alpha$ ) and IL-1 $\beta$. High levels of interleukin- 6 result in activation of $\mathrm{T}$ helper 17 that triggers severe inflammatory response in COVID-19 patients. Furthermore, the level of IL-6 has been related to the severity of COVID-19 infection and used as a predictor of clinical prognosis [9]. In addition, other inflammatory mediators such as interferons e.g., IFN- $\alpha$, and IFN- $\gamma$, interleukins e.g., IL-1 $\beta$, IL-12, IL-18, tumor necrosis factor- $\alpha$ (TNF- $\alpha$ ), and chemokines e.g., CCL2, CXCL10 have been reported in severely ill patients. These mediators represent the corner stone in developing cytokine storm that results in uncontrolled systemic inflammatory reaction with subsequent acute respiratory distress syndrome (ARDS), multiple organ failure and eventually death [10]. This mechanism of cell damage represents a target for already existing medications that modulate the immune response such as tocilizumab, monoclonal antibody against IL-6 receptors [11]. Based on its anti-inflammatory effects, colchicine has also gained attention to be utilized in the management of COVID-19 patient [12]. Colchicine is an alkaloid drug that is formed from a plant called "Colchicum autumnale", also named as "autumn crocus" [13]. Colchicine is used in many autoinflammatory conditions e.g., gout, familial Mediterranean fever, and Behçet's syndrome [14]. Colchicine exerts its anti-inflammatory effect through inhibition of formation of microtubules which is considered an essential step in several cellular processes such as division, signalling, and migration. Microtubule formation has been found to facilitate the invitro infection by coronavirus [15]. Also, colchicine affects the cytokine cascade by inhibiting IL-1 $\beta$ leading to reduction in neutrophils recruitment, free radicles production and inflammasome stimulation [13]. Moreover, it has been documented that colchicine has been used in cardiac conditions caused by viral infection e.g., myocarditis caused by CMV or EBV, interstitial pneumonia, pericarditis resulting from influenza B infection. Beside the fore mentioned effects of colchicine, its usage is considered safe and affordable with wide availability [16]. This raises the concerns about the effectiveness of colchicine in COVID-19 treatment and the possibility of providing an improvement of the clinical course of the disease.

In conclusion, colchicine could have an important role in the management of COVID-19. New randomized controlled clinical trials to assess the effectiveness of colchicine in the management of COVID-19 are strongly and urgently needed.

\section{References}

[1] Weiss SR, Leibowitz JL. Coronavirus pathogenesis. Adv Virus Res 2011;81:85-164.

[2] Zhong NS, Zheng BJ, Li YM, Poon LLM, Xie ZH, Chan KH, et al. Epidemiology and cause of severe acute respiratory syndrome (SARS) in Guangdong, People's Republic of China, in February, 2003. Lancet 2003;362(9393):1353-8.

[3] Moh Zaki A, Van Boheemen S, Bestebroer TM, Osterhaus ADME, Fouchier RAM. Isolation of a Novel Coronavirus from a Man with Pneumonia in Saudi Arabia. N Engl J Med 2012;367:1814-34.

[4] Wu F, Zhao S, Yu B, Chen Y-M, Wang W, Hu Y, et al. Complete genome characterisation of a novel coronavirus associated with severe human respiratory disease in Wuhan, China. bioRxiv 2020. doi: 10.1101/2020.01.24.919183

[5] Kuba K, Imai Y, Rao S, Gao H, Guo F, Guan B, Huan Y, Yang P, Zhang Y, Deng W, Bao L, Zhang B, Liu G, Wang Z, Chappell M, Liu Y, Zheng D, Leibbrandt A, Wada T, Slutsky AS, Liu D, Qin C, Jiang C, Penninger JM. A crucial role of angiotensin converting enzyme 2 (ACE2) in SARS coronavirus-induced lung injury. Nat Med 2005;11(8):875-9.

[6] Duan G. Intuition on virology, epidemiology, pathogenesis, and control of COVID-19. Nov Res Microbiol J 2020;4(5):955-67.

[7] Channappanavar R, Perlman S. Pathogenic human coronavirus infections: causes and consequences of cytokine storm and immunopathology. Semin Immunopathol 2017;39(5):529-39. 
[8] Tardif J, Bouabdallaoui N, Allier PLL. Efficacy of Colchicine in Non-Hospitalized Patients with COVID-19. medRxiv 2021. doi: 10.1101/2021.01.26.21250494.

[9] Zhang C, Wu Z, Li JW, Zhao H, Wang GQ. Cytokine release syndrome in severe COVID-19: interleukin-6 receptor antagonist tocilizumab may be the key to reduce mortality. Int J Antimicrob Agents 2020;55(5):105954.

[10] Coperchini F, Chiovato L, Croce L, Magri F, Rotondi M. The cytokine storm in COVID-19: An overview of the involvement of the chemokine/chemokine-receptor system. Cytokine Growth Factor Rev 2020;53:25-32.

[11] Liu B, Li M, Zhou Z, Guan X, Xiang Y. Can we use interleukin-6 (IL-6) blockade for coronavirus disease 2019 (COVID-19)-induced cytokine release syndrome (CRS)? J Autoimmun 2020;111:102452..

[12] Della-Torre E, Della-Torre F, Kusanovic M, et al. Treating COVID-19 with colchicine in community healthcare setting. Clin Immunol. 2020;217:108490.

[13] Dasgeb B, Kornreich D, McGuinn K, Okon L, Brownell I, Sackett DL. Colchicine: an ancient drug with novel applications. Br J Dermatol 2018;178(2):350-6.

[14] Terkeltaub RA. Colchicine Update: 2008. Vol. 38, Seminars in Arthritis and Rheumatism. W.B. Saunders; 2009. p. 411-9.

[15] Coronavirus H, Milewska A, Nowak P, Owczarek K, Szczepanski A, Zarebski M. Entry of Human Coronavirus NL63 into the Cell. J Virol 2018;92(3):1-16.

[16] Scarsi M, Piantoni S, Colombo E, Airó P, Richini D, Miclini M, et al. Association between treatment with colchicine and improved survival in a single-centre cohort of adult hospitalised patients with COVID-19 pneumonia and acute respiratory distress syndrome. Ann Rheum Dis 2020;79(10):1286-9. 